\title{
Video Article \\ Solid Phase Synthesis of a Functionalized Bis-Peptide Using "Safety Catch" Methodology
}

\author{
Conrad T. Pfeiffer ${ }^{1}$, Christian E. Schafmeister ${ }^{1}$ \\ ${ }^{1}$ College of Science and Technology, Temple University \\ Correspondence to: Christian E. Schafmeister at meister@temple.edu \\ URL: https://www.jove.com/video/4112 \\ DOI: doi:10.3791/4112
}

Keywords: Chemistry, Issue 63, bis-peptides, solid phase peptide synthesis, bis-amino acids, safety catch, HMBA, DTRA

Date Published: 5/15/2012

Citation: Pfeiffer, C.T., Schafmeister, C.E. Solid Phase Synthesis of a Functionalized Bis-Peptide Using "Safety Catch" Methodology. J. Vis. Exp. (63), e4112, doi:10.3791/4112 (2012).

\section{Abstract}

In 1962, R.B. Merrifield published the first procedure using solid-phase peptide synthesis as a novel route to efficiently synthesize peptides. This technique quickly proved advantageous over its solution-phase predecessor in both time and labor. Improvements concerning the nature of solid support, the protecting groups employed and the coupling methods employed over the last five decades have only increased the usefulness of Merrifield's original system. Today, use of a Boc-based protection and base/nucleophile cleavable resin strategy or Fmoc-based protection and acidic cleavable resin strategy, pioneered by R.C. Sheppard, are most commonly used for the synthesis of peptides ${ }^{1}$.

Inspired by Merrifield's solid supported strategy, we have developed a Boc/tert-butyl solid-phase synthesis strategy for the assembly of functionalized bis-peptides ${ }^{2}$, which is described herein. The use of solid-phase synthesis compared to solution-phase methodology is not only advantageous in both time and labor as described by Merrifield ${ }^{1}$, but also allows greater ease in the synthesis of bis-peptide libraries. The synthesis that we demonstrate here incorporates a final cleavage stage that uses a two-step "safety catch" mechanism to release the functionalized bis-peptide from the resin by diketopiperazine formation.

Bis-peptides are rigid, spiro-ladder oligomers of bis-amino acids that are able to position functionality in a predictable and designable way, controlled by the type and stereochemistry of the monomeric units and the connectivity between each monomer. Each bis-amino acid is a stereochemically pure, cyclic scaffold that contains two amino acids (a carboxylic acid with an a-amine) ${ }^{3,4}$. Our laboratory is currently investigating the potential of functional bis-peptides across a wide variety of fields including catalysis, protein-protein interactions and nanomaterials.

\section{Video Link}

The video component of this article can be found at https://www.jove.com/video/4112/

\section{Protocol}

\section{Setup}

1. The reaction set-up for the solid-phase synthesis is a polypropylene filter cartridge or glass reactor that is connected via polypropylene tubing to an enclosed filtering flask under vacuum as shown in Figure 1. The reaction can be mixed by a magnetic stir bar or by bubbling nitrogen through the reactor.

2. A gas manifold connected to an Argon cylinder equipped with a drying tube and oil bubbler is also recommended as it allows the reaction vessel to be contained under an inert atmosphere and allows the removal of reagents from sealed containers.

3. All operations are performed in a fume hood and proper personal protective equipment (safety glasses, lab coat and nitrile gloves) is required.

\section{Loading First Bis-Peptide Onto Resin}

1. Weigh $114 \mathrm{mg}$ of HMBA-AM Resin $(0.88 \mathrm{mmol} / \mathrm{g}$ loading, $100 \mu \mathrm{mol})$ into $8 \mathrm{~mL}$ reaction vessel and add magnetic stir bar. Cap the top of the vessel with a rubber septum and purge the tube with argon for at least 5 minutes.

2. In the meantime, weigh $117.3 \mathrm{mg}$ of compound 1 of Figure $3(586.63 \mathrm{~g} / \mathrm{mol}$, 2eq) and $59.2 \mathrm{mg}$ of 1-(mesitylene-2-sulfonyl)-3-nitro-1,2,4triazole (MSNT, $296.0 \mathrm{~g} / \mathrm{mol}$, 2eq) into a $15 \mathrm{~mL}$ disposable centrifuge tube and dissolve in $2 \mathrm{~mL}$ anhydrous dichloromethane (DCM). Add 24 $\mu \mathrm{L}$ of 1-methylimidazole (NMI, $80.81 \mathrm{~mL} / \mathrm{mol}$, 3eq) to solution and mix until completely dissolved.

3. Transfer the activated solution to the reaction vessel via syringe and allow to stir under argon overnight $(\sim 10$ hours).

4. Remove the septum and drain the reaction mixture. Wash the resin with DCM (5x) and dimethylformamide (DMF) (5x). Perform the "methyl red test" described in section 10.1 to assess the degree of resin loading. If the resin remains red during the methyl red test then steps 2.2 and 
2.3 should be repeated. A yellow color, indicative of a negative methyl red test, is preferred; however, since any remaining hydroxyl groups will be capped in the next step, a slightly positive result (light orange resin color) may be acceptable.

\section{Deprotection of First Bis-Peptide and Simultaneous Resin Capping}

1. Add $1 \mathrm{~mL}$ of $\mathrm{DCM}$ to the reaction vessel then add $1 \mathrm{~mL} 33 \%$ hydrogen bromide in acetic acid dropwise over 30 seconds (bubbling occurs) and allow to stir for 15 minutes. Drain and wash the resin with DCM $(5 x)$ then repeat process once more.

2. Wash the resin with DCM (5x) then DMF (5x). Neutralize the resin by washing twice with a $5 \%$ v/v solution of N,N-diisopropylethylamine (DIPEA) in DMF then wash with DCM (5x) and DMF (5x) again. Perform the "methyl red test" and "chloranil test" discussed in section 10.1 and 10.2. Results should be negative for the methyl red test and positive for the chloranil test.

\section{Coupling Boc/tBu-Protected Functionalized Bis-Amino Acid}

1. Reintroduce an inert atmosphere to the resin-containing reaction vessel by washing three times with anhydrous DCM then attach a septum and argon line. Purge and wash the vessel by adding 1-2 $\mathrm{mL}$ of anhydrous DCM and letting stir for 30 seconds then draining the vessel until the argon line bubbler begins to rise. Do this at least once more.

2. Prepare a solution of $0.15 \mathrm{M}$ functionalized bis-amino acid (3eq) and $245 \mathrm{mg}$ of 1-hydroxy-7-azabenzotriazole (HOAt, $136.11 \mathrm{~g} / \mathrm{mol}$, $18 \mathrm{eq})$ in $2 \mathrm{~mL}$ of 2:1 DCM:DMF in a flame dried test tube under argon atmosphere. Add $47 \mathrm{\mu L}$ of diisopropylcarbodiimide (DIC, $156.6 \mathrm{~mL} / \mathrm{mol}, 3 \mathrm{eq})$ and stir for 90 minutes.

3. Add $35 \mu \mathrm{L}$ DIPEA ( $174.19 \mathrm{~mL} / \mathrm{mol}, 2 \mathrm{eq})$ in $666 \mu \mathrm{L}$ anhydrous DMF to resin and allow to stir for 5 minutes.

4. Transfer the pre-activated bis-amino acid solution to reaction vessel via syringe and allow to stir overnight.

5. Drain the reaction mixture and wash twice with anhydrous DCM while under argon.

6. To promote the closure of the diketopiperazine, add a $0.25 \mathrm{M}$ solution of HOAt $(136.11 \mathrm{~g} / \mathrm{mol}, 10 \mathrm{eq})$ and DIC $(156.6 \mathrm{~mL} / \mathrm{mol}, 10 \mathrm{eq})$ in a 4 $\mathrm{mL}$ of 1:1 DCM:DMF and allow to stir under argon for 1 hour.

7. Remove the septum and drain the reaction mixture. Wash the resin with DCM (5x) and DMF (5x). If desired, perform the "chloranil test" discussed in section 10.2

\section{Deprotection of Boc/tBu-Protected Functionalized Bis-Amino Acid}

1. Add $2 \mathrm{~mL}$ of a solution of 95:5 trifluororacetic acid (TFA):triisopropylsilane (TIPS) to the reaction vessel and allow it to stir for 1 hour. Drain and wash the resin for about 30 seconds with DCM $(5 x)$ then repeat process once more.

2. Wash the resin with DCM (5x) then DMF (5x). Neutralize the resin by washing twice with a $5 \% \mathrm{v} / v$ solution of DIPEA in DMF then wash DCM $(5 x)$ and DMF $(5 x)$ again. If desired, perform the "chloranil test" discussed in section 10.2 .

\section{Repeat steps 4 and 5 as desired to synthesize targeted bis-peptide.}

\section{Functionalizing the Bis-Peptide Prolidine End}

1. The prolidine end of the growing bis-peptide may be acylated independently or together through a diketopiperazine. Also, this end can be left protected, which will be cleaved latter, affording the free amino acid. If desired, perform the "chloranil test" discussed in section 10.2 to assess coupling efficiency.

\section{Deprotection of Fmoc and Acylation of the Quaternary End of the Bis-Peptide}

1. A $2 \mathrm{~mL}$ solution of $20 \%$ piperidine in DMF is added and the reaction is mixed for 20 minutes. Drain and wash the resin with $\mathrm{DMF}(5 \mathrm{x})$ then repeat process once more.

2. Wash the resin with DCM $(5 x)$ then DMF $(5 x)$.

3. Prepare a $0.15 \mathrm{M}$ solution of amino acid (3eq) in $2 \mathrm{~mL}$ of $\mathrm{N}$-methylpyrrolidone (NMP) with $114 \mathrm{mg}$ 2-(7-aza-1H-benzotriazole-1-yl)-1,1,3,3tetramethyluronium hexafluorophosphate (HATU, $380.2 \mathrm{~g} / \mathrm{mol}, 3 \mathrm{eq}$ ) and $104.5 \mu \mathrm{L}$ DIPEA $(174.19 \mathrm{~mL} / \mathrm{mol}, 6 \mathrm{eq})$ and $\mathrm{mix}$ well. Add to reaction vessel and allow to stir for 6 hours.

4. Wash the resin with DCM $(5 x)$ then DMF $(5 x)$

\section{Remove the Boc Group from the Resin Bound Amino Acid and Cleave from Resin}

1. Add $2 \mathrm{~mL}$ of a 1:1 TFA:DCM solution to the reaction vessel and allow to stir for 30 minutes. Drain and wash the resin with $\mathrm{DCM}$ (5x) then repeat process once more.

2. Wash and drain the resin for 30 seconds with DCM (5x) then DMF $(5 x)$.

3. Add $2 \mathrm{~mL}$ of a solution of $10 \%$ DIPEA in anhydrous DMF and allow to stir $24-48$ hours.

4. Collect reaction mixture into pre-weighed round bottom flask. Transfer $30 \mu \mathrm{L}$ of this solution to $450 \mu \mathrm{L}$ of THF in an LC-MS vial and submit for analysis. Wash the resin with additional aliquots of DMF and collect into the round bottom flask then remove solvent in vacuo. 


\section{Purification of Bis-Peptide}

1. Dissolve crude bis-peptide in a minimal amount of dimethyl sulfoxide $(100-250 \mu \mathrm{L})$ and transfer into HPLC vial insert. Place insert into autosampler of semi-prepitive HPLC system (Hewlett Packard 1100 Series) equipped with an XTerra Prep MS C18 5 m $7.8 \times 150$ mm column and a $100 \mu \mathrm{L}$ injection loop.

2. Perform multiple $50 \mu \mathrm{L}$ injections of the sample using a gradient program of $5-95 \%$ acetonitrile in water with $0.1 \%$ formic acid over 30 minutes while monitoring at $274 \mathrm{~nm}$. Collect the product peak in a pre-weighed disposable centrifuge tube and freeze dry using a lyophilizer. Caution should be taken with the first run as a slight shift in peak retention time compared to analytical LCMS is typically observed.

\section{Assessment Methods}

1. METHYL RED TEST ${ }^{7}$ : Remove $\sim 1 \mathrm{mg}$ of dry resin via disposable pipette and rinse into $4 \mathrm{~mL}$ reaction vessel. Add a solution of $20 \mathrm{mg}$ methyl red, $50 \mu \mathrm{L}$ N,N'-diisopropylcarbodiimide (DIC), and $5 \mathrm{mg}$ 4-dimethylaminopyridine (DMAP) in $500 \mu \mathrm{L}$ anhydrous DCM and allow to stir for 5-10 min. Drain and wash resin with DCM until the filtrate becomes colorless. Positive indication is the resin beads remaining orange or red.

2. CHLORANIL TEST ${ }^{12}$ : Transfer $\sim 1 \mathrm{mg}$ of dry resin into a small vial via disposable pipette. Add 3 drops of both a $0.8 \mathrm{mM}$ chloranil in DMF solution and $2 \%$ acetaldehyde in DMF solution and let sit at room temperature for 5-10 minutes. Positive indication is the resin beads turning blue/purple.

3. ACTIVATION TRAP TEST: Activated compounds during the synthesis can be assessed by transferring a small amount (5-10 $\mu \mathrm{L})$ of the activated solution to a liquid chromatography-mass spectrometry (LC-MS) vial containing $50 \mu \mathrm{L}$ of pyrrolidine. Mix by hand for a few seconds (solution should become yellow) then dilute with $450 \mu \mathrm{L}$ of tetrahydrofuran (THF) and submit for LC-MS analysis.

4. ANALYTICAL LC-MS: The final product and activated intermediates can be assessed using an HP 1200 series LC-MS system equipped with a Waters Xterra MS C18 $3.5 \mu \mathrm{m} 4.6 \mathrm{~mm} \times 150 \mathrm{~mm}$ column and a gradient system of 5-95\% acetonitrile in water with $0.1 \%$ formic acid over 30 minutes.

\section{Representative Results}

An example of both crude (Figure 4) and purified (Figure 5) LCMS traces are provided. Purified yields of approximately $10 \%$ are expected using the methods outlined above.

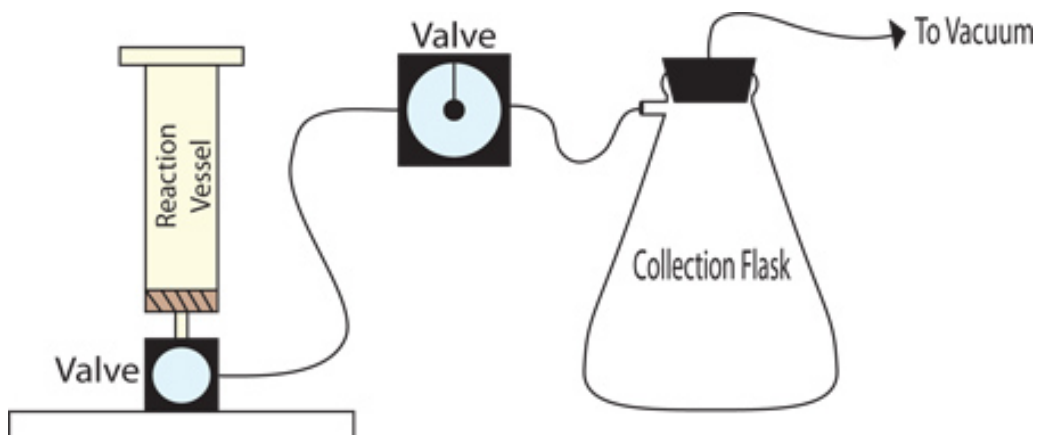

Magnetic

Stir Plate

Figure 1. Diagram of Experimental Set-Up for Solid Phase Synthesis. 


\section{Quaternary}

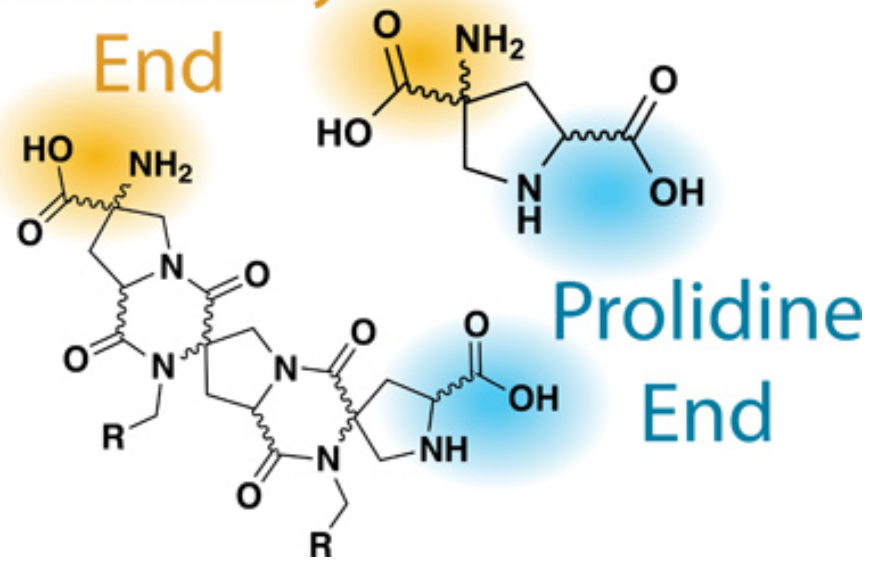

Figure 2. Relevant Nomenclature of Bis-Amino Acids/Bis-Peptides.
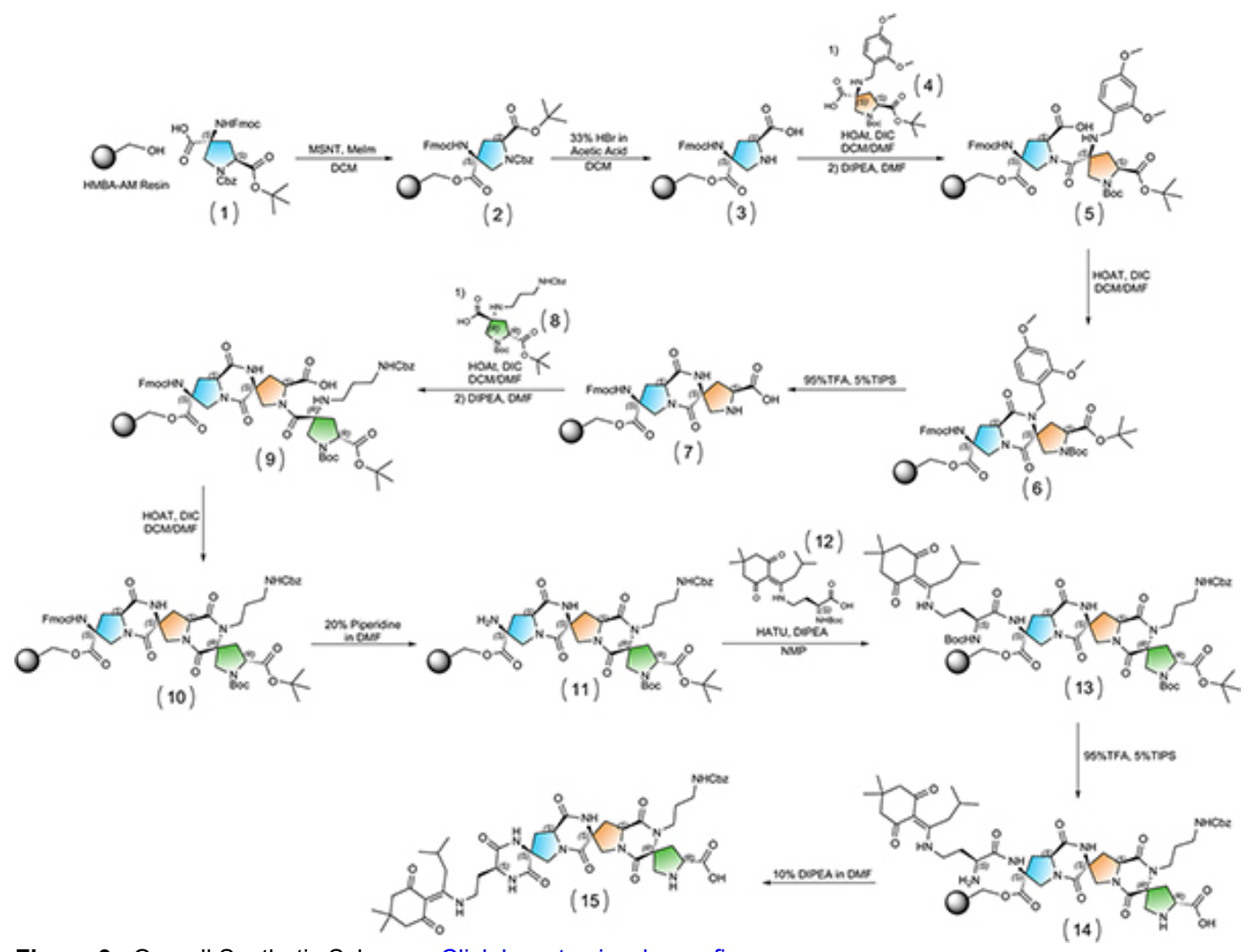

Figure 3. Overall Synthetic Scheme. Click here to view larger figure. 


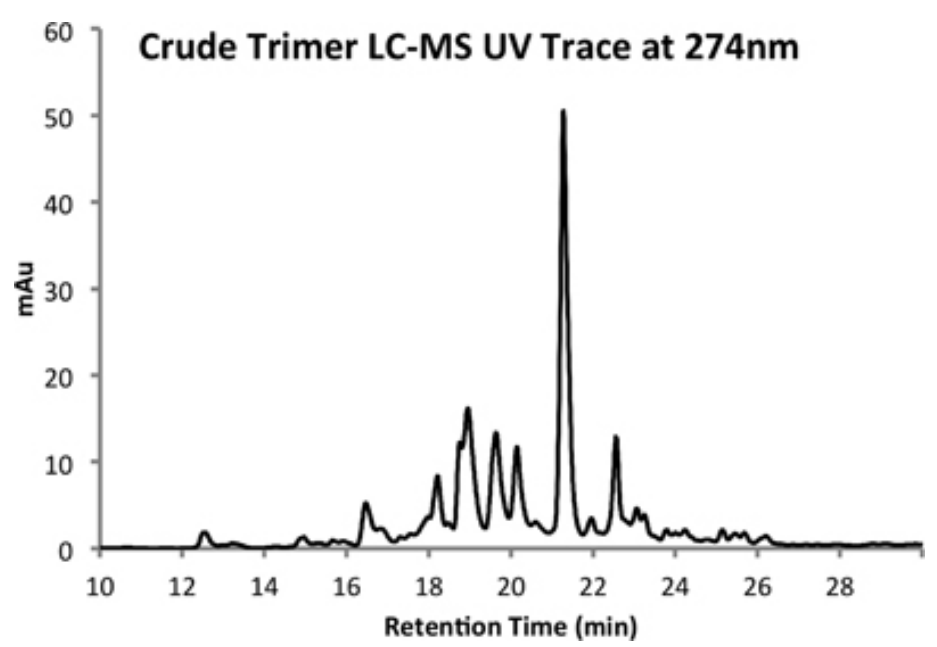

Figure 4a. HPLC Trace of Crude Product at $274 \mathrm{~nm}$.

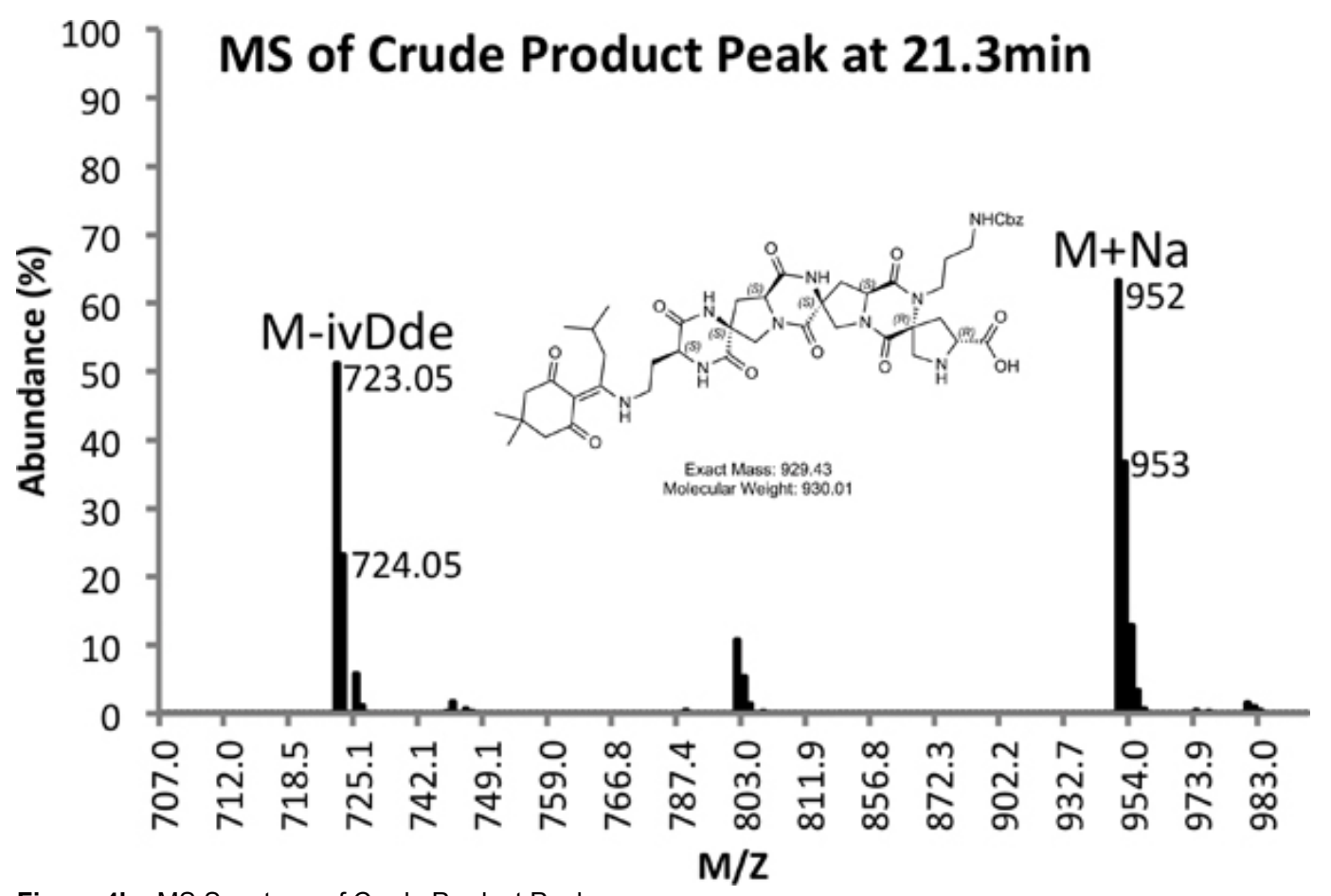

Figure 4b. MS Spectrum of Crude Product Peak.

\section{Purified Trimer LC-MS UV Trace at 274nm}

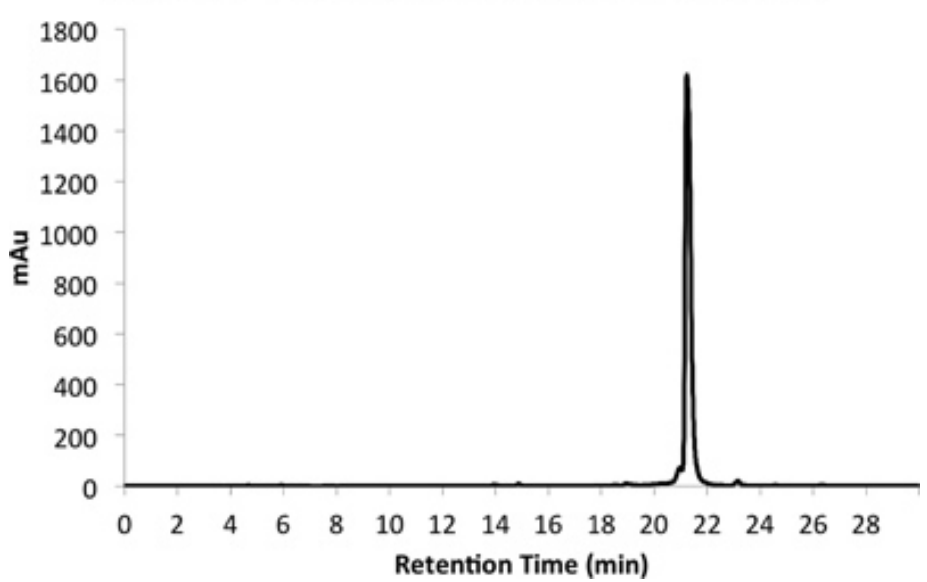


Figure 5a. HPLC Trace of Purified Product at $274 \mathrm{~nm}$.

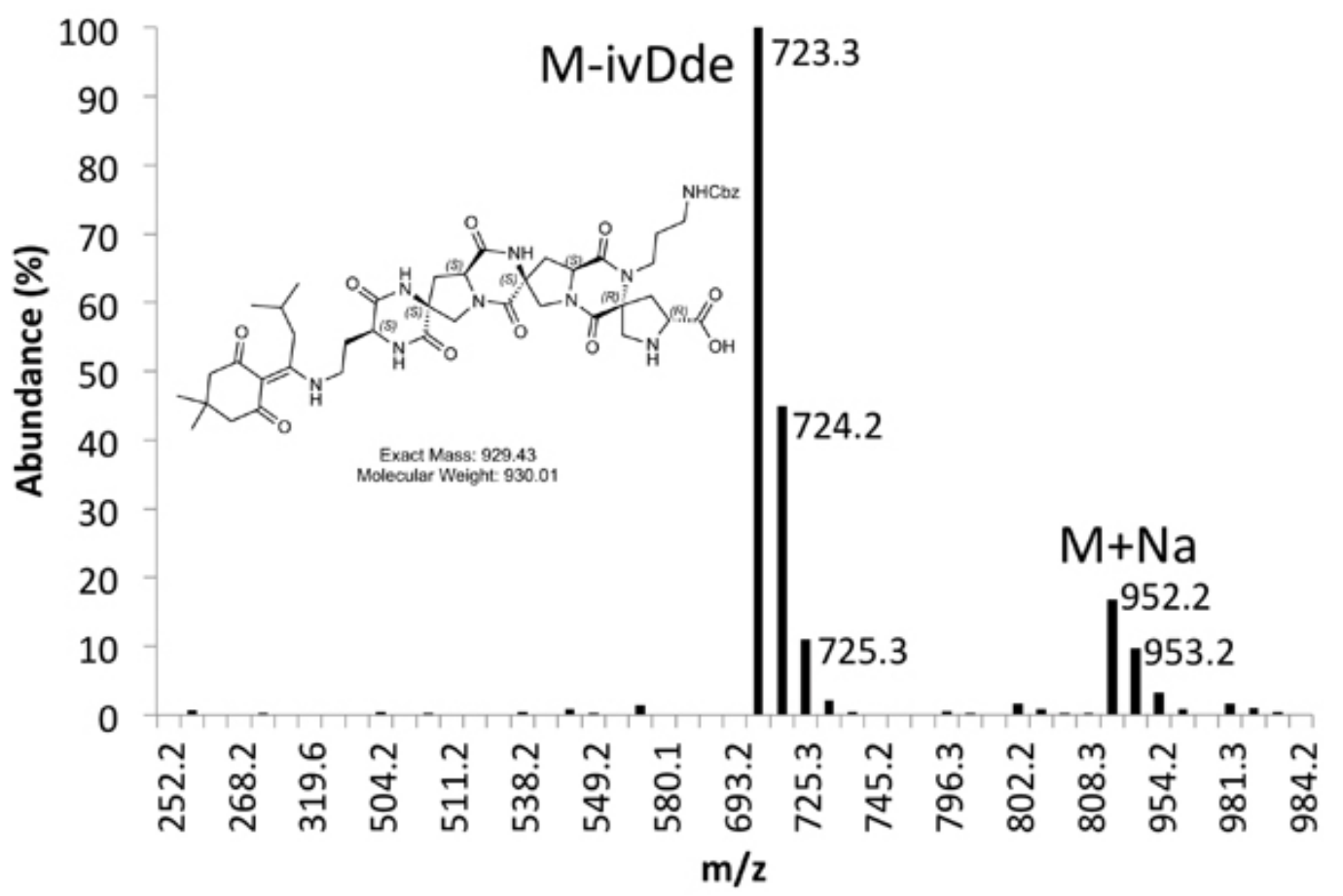

Figure 5b. MS Spectrum of Purified Product Peak.

\section{Discussion}

The synthetic approach presented herein provides a method for the synthesis of functionalized bis-peptides from bis-amino acid building blocks using common solid-phase peptide synthesis techniques. The monomer synthesis of these "Pro4" building blocks from trans-4-hydroxyproline ${ }^{3}$ is highly scalable and has been successfully completed to the hydantoin stage at a $600 \mathrm{mmol}(234 \mathrm{~g})$ scale (unpublished). Once the monomers are in hand, the use of solid-phase techniques provides a more rapid method of bis-peptide synthesis than our current solution-phase methodology ${ }^{4}$ by eliminating the need for reaction work-ups and intermediate purifications.

The primary challenge in solid-phase synthesis is diagnosing synthetic progress and problem solving since no intermediates are isolated. This has lead to the development of many colorimetric tests including those to identify if free amines (Kaiser Test ${ }^{10}$ ) or free hydroxyls (Methyl Red Test $^{7}$ ) are exposed on resin. Unfortunately, the commonly used Kaiser Test ${ }^{10}$ is not generally applicable in our solid-phase synthesis due to the almost exclusive use of secondary amines or amines attached to a quaternary carbon. Other options for assessment on HMBA resin include test cleavages using a nucleophile such as hydrazine ${ }^{11}$, quantitative Fmoc cleavage monitored by UV/Vis ${ }^{1,11}$, and trapping and analyzing incoming activated compounds.

Another overlooked issue in solid-phase synthesis is the repetitive nature of synthetic steps required by the operator. With this is mind, the authors strongly recommend the use of a spreadsheet or checklist when performing any manual solid-phase peptide synthesis.

The difficultly in using bis-peptides for solid-phase synthesis compared to common a-amino acids includes the potential for more difficult couplings due to steric hindrance, the need for on-resin diketopiperazine closures, and simultaneous deprotections $(\mathrm{Boc} / \mathrm{tBu}$; $\mathrm{Cbz} / \mathrm{tBu})$. Another difficultly lies in achieving quantitative release from the resin using this "safety catch" method when compared to more conventional means. With these factors in mind, it is very possible that further optimization of this method can be achieved and current efforts are underway in our group to improve the method presented here.

\section{Disclosures}

Production and Free Access to this article is sponsored by the United States Defense Threat Reduction Agency.

\section{Acknowledgements}

The authors would like to thank Dr. Zachary Z. Brown and Jennifer Alleva for the initial development of this solid phase synthesis technique and Matthew F.L. Parker for helpful discussions. This work is supported by the Defense Threat Reduction Agency (DOD-DTRA) (HDTRA1-09-1-0009) and the Horst Witzel Fellowship Award supported by Cephalon, Inc. 


\section{References}

1. Atherton, E. \& Sheppard, R.C. Solid Phase Peptide Synthesis: A Practical Approach. Oxford University Press., (1989).

2. Brown, Z.Z., Alleva, J., \& Schafmeister, C.E. Solid-Phase Synthesis of Functionalized Bis-Peptides. Biopolymers. 96, 578-585 (2010).

3. Schafmeister, C.E., Brown, Z.Z., \& Gupta, S. Shape-Programmable Macromolecules. Acc. Chem. Res. 41, 1387-1398 (2008).

4. Brown, Z.Z. \& Schafmeister, C.E. Synthesis of Hexa- and Pentasubstituted Diketopiperazines from Sterically Hindered Amino Acids. Org. Let. 12, 1436-1439 (2010).

5. Nielson, J. \& Lyngso, L.O. Combinatorial Solid-Phase Synthesis of Balanol Analogues. Tet. Lett. 37, 8439-8442 (1996).

6. Blankemeyer-Menge, B., Nimtz, M., \& Frank, R. An Efficient Method for Anchoring Fmoc-Amino Acids to Hydroxyl-Functionalized Solid Supports. Tet. Lett. 31, 1701-1704 (1990).

7. Komba, S., Sasaki, S., \& Machida, S. A New Colorimetric Test for Detection of Hydroxyl Groups in Solid-Phase Synthesis. Tet. Lett. 48, 2075-2078 (2007).

8. Demner, O. Dijkgraaf, I. Schottelius, M. Wester, H.J., \& Kessler, H. Introduction of Functional Groups into Peptides via N-Alkylation. Org. Lett. 10, 2015-2018 (2008).

9. Van der Plas, S.E., Van Hoeck, E. Lynen, F., Sandra, P., \& Madder, A. Toward a New SPE Material for EDCs: Fully Automated Synthesis of a Library of Tripodal Receptors Followed by Fast Screening by Affinity LC. Eur. J. Org. Chem. 11, 1796-1805 (2009).

10. Kaiser, E., Colescot, R.L., Bossinger, C.D., \& Cook, P.I. Color Test for Detection of Free Terminal Amino Groups in Solid-Phase Synthesis of Peptides. Anal. Biochem. 34, 595-598 (1970).

11. Chan, W.C. \& White, P.D. Fmoc Solid Phase Peptide Synthesis: A Practical Approach. Oxford University Press., (2000).

12. Vojkovsky, T. Detection of Secondary Amines on Solid-Phase. Peptide Research. 71, 236-237 (1995). 